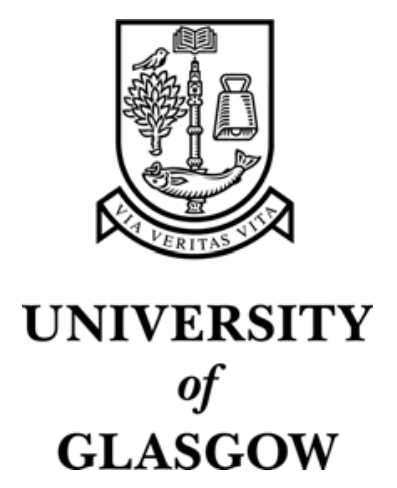

Watling, J.R. and Barker, J.R. and Asenov, A. (2000) Soft sphere model for electron correlation and scattering in the atomistic modelling of semiconductor devices. In, Barker, J.R. and Watling, J.R., Eds.

International Workshop on Computational Electronics, 22-25 May 2000, pages pp. 159-160, Glasgow, UK.

http://eprints.gla.ac.uk/3021/ 


\title{
Soft sphere model for electron correlation and scattering in the atomistic modelling of semiconductor devices
}

\author{
J. R. Watling, J. R. Barker ${ }^{*}$ and A. Asenov \\ Nanoelectronics Research Centre \\ Department of Electronics and Electrical Engineering \\ University of Glasgow, Glasgow G12 8LT, Scotland, UK
}

*Tel: +44 141330 5221; Fax: +44 141330 4907; E-mail: jbarker@elec.gla.ac.uk

The atomistic modelling of silicon MOSFET devices becomes essential at deep sub-micron scales when it is no longer possible to represent the charged impurities by a continuous charge distribution with a determined doping density. Instead the spatial distribution and the actual number of dopants must be treated as discrete random variables. This regime has been modelled recently by a number of groups [1-3] using a low voltage approximation where the current flow is treated in a drift-diffusion or generalised drift diffusion approximation. Here the current flow is determined from current continuity via the solution to the Poisson equation for the potential in the presence of a static discrete impurity distribution. Among the established predictions, we may mention the strong fluctuations in threshold voltage from device to device where the macroscopic device parameters, doping density and spatial dimensions, are identical.

These atomistic models should also, for self-consistency, treat the channel electrons as a discrete spatial distribution which will also fluctuate in number. This is an exceptionally difficult problem to solve on the atomistic scale because the details of the injection and extraction of carriers at source and drain requires consideration and indeed the time scale on which the statistics are computed becomes important when we consider that the discrete carriers are mobile. Furthermore to obtain useful statistics on the variance of the device characteristics (now treated as random variables) it becomes necessary to model many thousands of macroscopically identical devices with different atomistic configurations. For this purpose the present paper addresses the issue of modelling the dynamics of discrete carrier flow in a semiconductor device utilising a simple model of the carrier-carrier scattering and carrier-fixed impurity scattering which is suitable for efficient simulations of large ensembles of devices.

Although we could in principle resort to direct Monte Carlo simulation of the full Coulomb interaction, this procedure is computationally prohibitive for ensembles of devices. Instead we represent the Coulomb potential (Fig. 1) by a soft-sphere model in which the potential is zero for the inter-carrier separation $r>2 R_{c}$ but jumps discontinuously to a constant value $W$ for $r \leq 2 R_{c}$ where $R_{c}$ and $W$ are to be regarded as adjustable parameters for the model, although physically they might be interpreted as the screening radius and Coulomb potential at twice the screening radius. In a classical collision model carriers undergo hard sphere scattering for energies less than $\mathrm{W}$, but pass through each others positions for higher energies (Fig. 2). The model is furthermore restricted to dilute collisions: only collision pairs are considered. This model has been investigated by Monte Carlo simulation to determine both its efficiency and accuracy for 
device simulation in the atomistic limit and results are presented for the carrier spatial correlation that ensues with and without fixed discrete dopant distributions (Fig 3a,b). Consequences for device ensemble modelling are discussed.

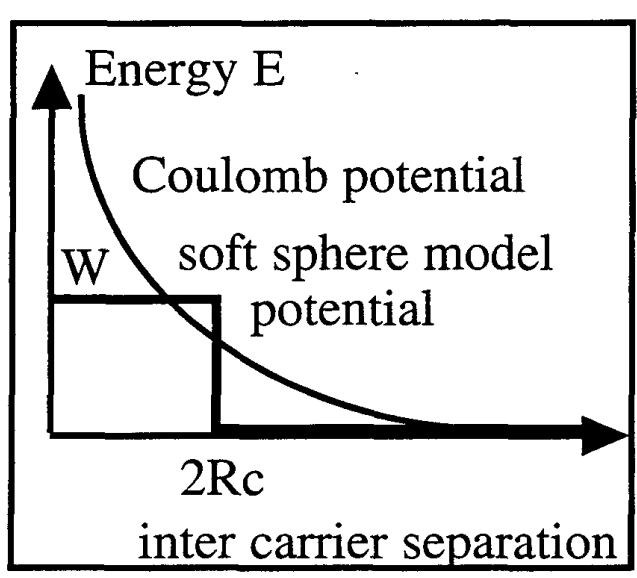

Figure. 1: Soft sphere scattering model potential

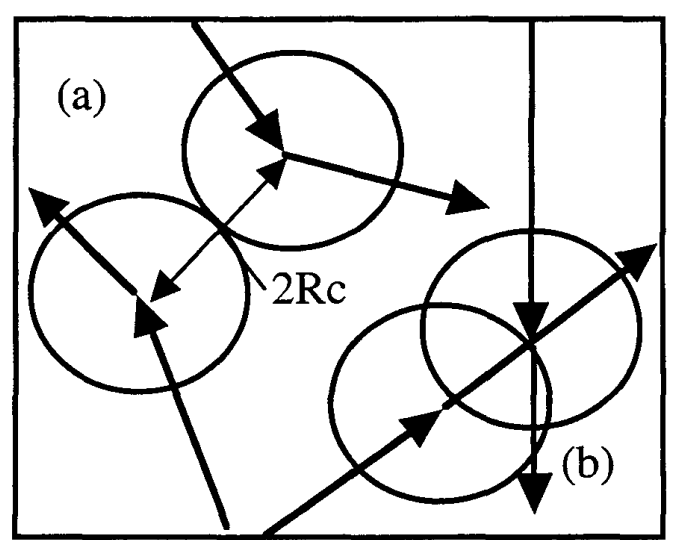

Figure 2: Carrier-carrier scattering in a soft sphere interaction potential (a) Energy $\mathrm{E}<\mathrm{W}$; (b) Energy E $>$ W.

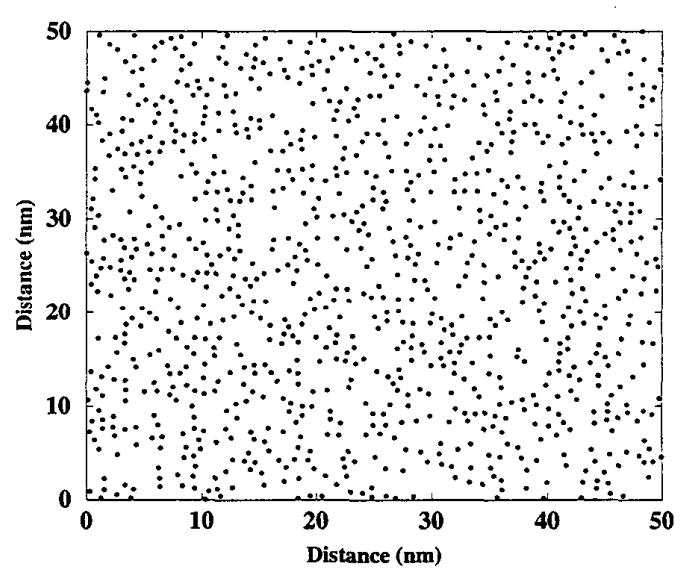

Fig.3b. Spatial correlation in a carrier ensemble due to soft sphere collisions

\section{References}

1. A. Asenov, Extended Abstracts IWCE-6, IEEE Cat 98EX116, 263 (1998).

2. D. Vasileska, W. J. Gross and D. K. Ferry, Extended Abstracts IWCE-6, IEEE Cat 98EX116, 259 (1998).

3. A. Asenov IEEE Trans. Electron Dev, 45, No 12, 2505 (1998). 